\title{
Experimental Study on PEDOT: PSS Conductive Polymer and N-doped Graphene Quantum Dots for $\mathrm{H}_{2} \mathrm{O}_{2}$ Sensing
}

\author{
Mahdieh Hakimi, ${ }^{*}$ Alireza Salehi, Farhad Akbari Boroumand \\ Department of Electronics, Faculty of Electrical Engineering, K. N. Toosi University of Technology, \\ 16315-1355, Tehran, Iran
}

\begin{abstract}
The detection of hydrogen peroxide $\left(\mathrm{H}_{2} \mathrm{O}_{2}\right)$ is considered important in various fields. This work described a resistive $\mathrm{H}_{2} \mathrm{O}_{2}$ sensor without using an enzyme, based on poly(3,4-ethylenedioxythiophene):poly(styrenesulfonate) (PEDOT:PSS) and N-doped graphene quantum dots (N-GQDs) composite on a transparent and flexible substrate. Using the drop-cast method, the uniform film was deposited onto interdigitated aluminum electrodes. The mechanism of $\mathrm{H}_{2} \mathrm{O}_{2}$ detection and reason for the use of GQDs have been explained. On exposure to $9 \mathrm{M} \mathrm{H}_{2} \mathrm{O}_{2}$, the sensing response obtained was $27.54 \%$.
\end{abstract}

Keywords : PEDOT:PSS, $\mathrm{N}-G Q D s, \mathrm{H}_{2} \mathrm{O}_{2}$

\section{Introduction}

Sensors are applied in many fields including environmental control, industrial production, food process control and medicine [1], [2]. Recently, conductive polymers have been used for the fabrication of various sensors, for example detection of hydrogen peroxide $\left(\mathrm{H}_{2} \mathrm{O}_{2}\right)$ uusually produced in enzymatic reactions [3]. $\mathrm{H}_{2} \mathrm{O}_{2}$ is not only produced in this way but also as a mediator in food, pharmaceutical, industrial, environmental analyses and other fields [4]. $\mathrm{H}_{2} \mathrm{O}_{2}$ detection is used to assess the safety and quality of pharmaceutical and cosmetic formulations [5]. Many methods have been used for $\mathrm{H}_{2} \mathrm{O}_{2}$ determination such as spectrometry [6], chemiluminescence [7], and electrochemical technique based on horseradish peroxidase (HRP) enzyme [8]. Since materials used for immobilization of enzymes may prevent electron transfer [9], thus in this study, a $\mathrm{H}_{2} \mathrm{O}_{2}$ sensor was designed that does not use enzyme and its immobilization. Poly(3,4-ethylenedioxythiophene):poly(styrenesulfonate) (PEDOT:PSS) is one of the conductive polymers that has been investigated for use as chemical and biological sensors [10], [11], [12]. Graphene composites with conductive polymers could enhance

\footnotetext{
* Corresponding author: Mahdieh Hakimi

Email: $\underline{\text { m.hakimi70@yahoo.com }}$
} 
sensing properties due to superior conductivity, high stability and good biocompatibility [4]. To make the use of graphene easier in nanodevices, an offer is the conversion of two-dimensional (2D) graphene to OD graphene quantum dots (GQDs) [13]. The dispersion of GQDs in common solvents is one of its advantages in various solutionprocessable applications [14]. GQDs are considered due to low cost [15], low toxicity and environmental compatibility [16]. Nitrogen substitution in the GQDs lattice produces more active sites which can be used in various fields including sensors [17]. This study is the first to assess the use of PEDOT:PSS and N-doped GQDs (N-GQDs) composite, as a resistive $\mathrm{H}_{2} \mathrm{O}_{2}$ sensor. In fact, this sensor shows the presence of $\mathrm{H}_{2} \mathrm{O}_{2}$ by film resistance change.

\section{Experimental}

In this study, N-GQDs were synthesized by hydrothermal processing. The details of synthesis of the N-GQDs is explained in a previous publication by the authors [18]. The PEDOT:PSS aqueous solution (1.3 wt $\%$ dispersed in $\mathrm{H}_{2} \mathrm{O}$, conductive grade) was purchased from Sigma-Aldrich. The N-GQD solution was combined with $50 \mathrm{wt} \%$ PEDOT:PSS solution and a homogeneous dispersion was obtained after stirring for $2 \mathrm{~h}$. this dispersion was considered as the sensing film.

Polyethylene terephthalate (PET) was used as a flexible substrate. Aluminum interdigitated electrodes with a width of $200 \mu \mathrm{m}$ were deposited on the substrate. Thereafter, $6 \mu \mathrm{L}$ N-GQDs/PEDOT:PSS composite was drop casted on the electrodes. After drying in the oven, the sensing film thickness was about $3 \mu \mathrm{m}$. Finally, the sensor was placed in the test chamber and its resistance was measured. A schematic picture of the fabricated sensor is shown in Fig. 1.

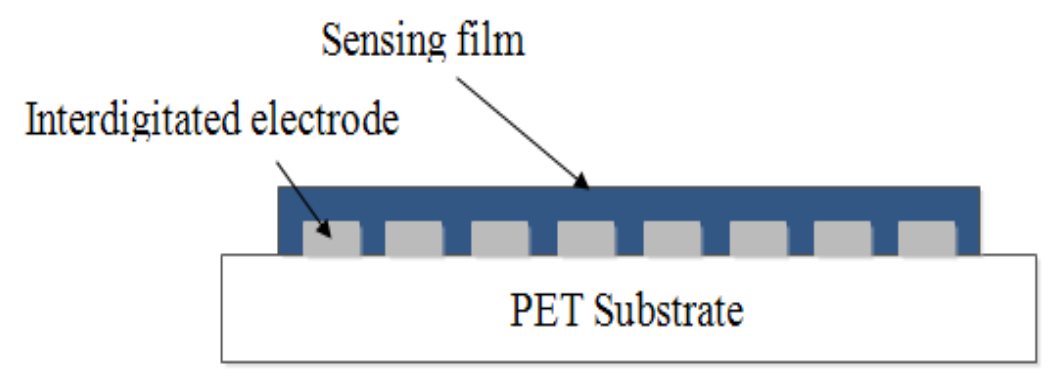

Fig. 1 : $\quad$ Schematic picture of the sensor structure. 


\section{Results and Discussion}

Fourier transform infrared spectroscopy (FT-IR) of N-GQDs/PEDOT:PSS is shown in Fig. 2. The bands at $3452.6,1638.23$ and $1261.9 \mathrm{~cm}^{-1}$ are attributed to the stretching and bending vibration of $-\mathrm{OH}$ groups, $\mathrm{C}=\mathrm{C}$ stretching and $\mathrm{C}-\mathrm{O}-\mathrm{C}$ stretching vibration, respectively. The $\pi$-electrons exist in both N-GQDs and PEDOT:PSS. This suggests the presence of $\pi-\pi$ interactions between them $[16,19]$.

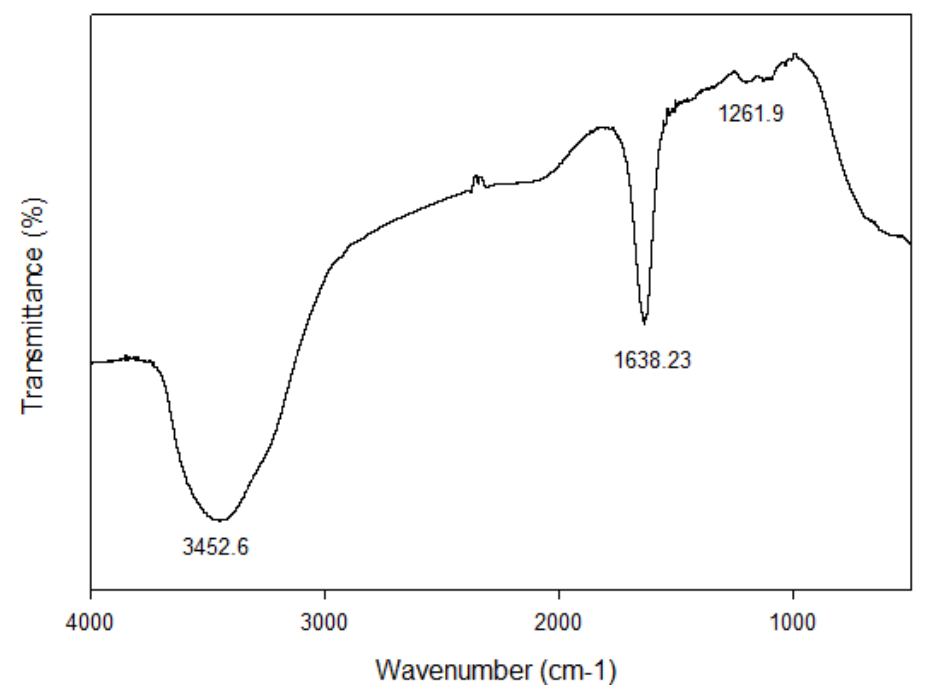

Fig. 2 : FTIR spectra of N-GQDs/PEDOT:PSS.

Upon exposure to $9 \mathrm{M} \mathrm{H}_{2} \mathrm{O}_{2}$, the N-GQDs/PEDOT:PSS sensor resistance increased from 2.07 to $2.64 \mathrm{k} \Omega$. PEDOT:PSS was reported as a resistive humidity sensor by Taccola et al. [20], who observed that the resistivity of PEDOT:PSS increased exposure to moisture, it relates to the swelling of the polymer (PSS) by absorption of water. Graphene/PEDOT:PSS as an ammonia detector has also been reported, and it has been shown that the swelling process occurs in the presence of this gas [19]. Swelling causes a reduction in connection between PEDOT chains (acting the role of conduction in PEDOT:PSS), reduces electron hopping and increases sensor resistance [19], [20]. Also, alcohols (e.g. methanol, ethanol and etc.) prevented the electrical connection between the PEDOT islands [21], [22].

All the gases and vapors contained above are the same, in terms of containing hydrogen. Therefore, one proposed mechanism of $\mathrm{H}_{2} \mathrm{O}_{2}$ detection is the separation of conductive pathways (including $\mathrm{N}-$ GQDs and PEDOT) as a result of the placement of $\mathrm{H}_{2} \mathrm{O}_{2}$ 
molecules between them as a screen (Fig. 3). Therefore, the resistance of the sensing film increases.
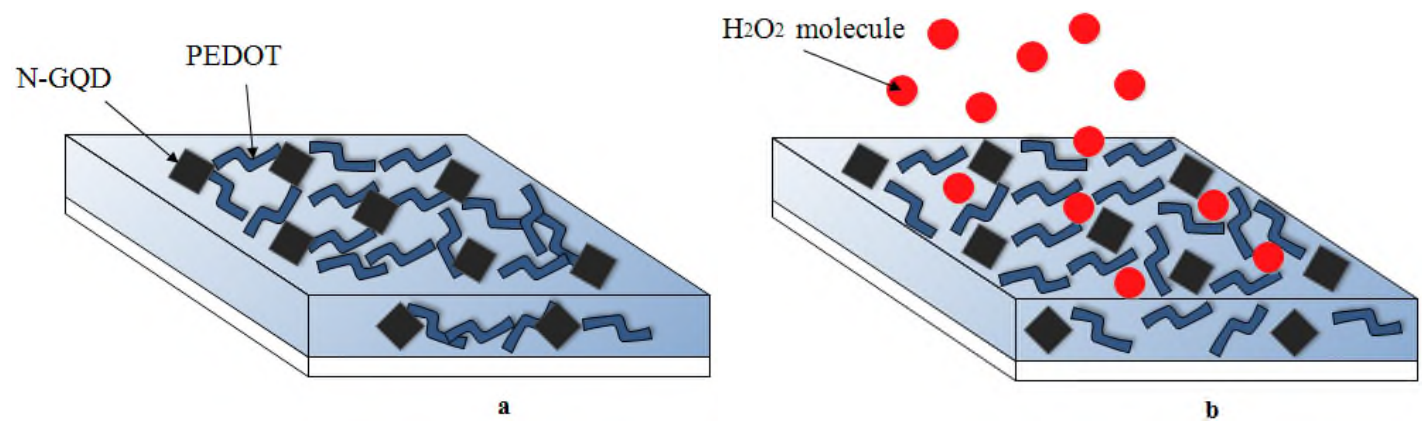

Fig. 3 : $\quad$ Schematic picture of the sensor (a) before and (b) after exposure to $\mathrm{H}_{2} \mathrm{O}_{2}$.

It is known that hydrogen is a reducing gas and donates electrons to the sensing film during adsorption. The electrons recombine with the holes and increase the resistance of a p-type semiconductor [23], [24]. Since $\mathrm{H}_{2} \mathrm{O}_{2}$ contains hydrogen, it is expected that a reduction/oxidation reaction occurs between PEDOT:PSS and $\mathrm{H}_{2} \mathrm{O}_{2}$. It can be another possible mechanism for $\mathrm{H}_{2} \mathrm{O}_{2}$ sensing which is in accordance with the reaction given in Eq. (1) [25]:

$$
\mathrm{H}_{2} \mathrm{O}_{2} \rightarrow \mathrm{O}_{2}+2 \mathrm{H}^{+}+2 e^{-}
$$

Since the sensing film is a p-type material, the carrier concentration decreases when the electrons of $\mathrm{H}_{2} \mathrm{O}_{2}$ analyte interact with the holes of sensing film. Therefore, the electrical resistance increases.

Graphene has a large surface area and thus, enhances the sensing response of the sensor [26] which is calculated with the equation:

Sensing response $\%=\frac{\left(R_{\mathrm{H}_{2} \mathrm{O}_{2}}-R_{\text {air }}\right)}{R_{\text {air }}} \times 100$

where $R_{\text {air }}$ and $R_{\mathrm{H}_{2} \mathrm{O}_{2}}$ are the sensor resistance in pure air and in the presence of $\mathrm{H}_{2} \mathrm{O}_{2}$, respectively. The sensing response of the N-GQDs/PEDOT:PSS sensor was obtained as $27.54 \%$, on exposure to $9 \mathrm{M} \mathrm{H}_{2} \mathrm{O}_{2}$. The advantages of this sensor include good sensing response, low cost and simplicity of manufacturing process and measurement.

Moreover, the larger surface area of graphene causes the reaction to be faster and easier between the sensing film and analyte, so the response time decreases [27]. The response time obtained was $480 \mathrm{~s}$ for the N-GQDs/PEDOT:PSS sensor and it is expected to decrease as the sensing film thickness decreases [28]. 


\section{Conclusions}

In summary, this study describes a $\mathrm{H}_{2} \mathrm{O}_{2}$ sensor without using enzyme. The sensor was fabricated by PEDOT:PSS conductive polymer and N-GQDs. It showed a response of $27.54 \%$ upon exposure to $9 \mathrm{M} \mathrm{H}_{2} \mathrm{O}_{2}$ for $480 \mathrm{~s}$. The advantages of this sensor include good sensing response, easy fabrication, flexibility, low cost and biocompatibility. In addition, reduction reaction and swelling process were explained as possible mechanisms for $\mathrm{H}_{2} \mathrm{O}_{2}$ sensing.

\section{References}

[1] Hopkins, A.R. and Lewis, N.S. (2001). Detection and classification characteristics of arrays of carbon black/organic polymer composite chemiresistive vapor detectors for the nerve agent simulants dimethylmethylphosphonate and diisopropylmethylphosponate. Analytical chemistry, vol. 73, no. 5, p. 884-892.

[2] Capone, S., Forleo, A., Francioso, L., Rella, R., Siciliano, P., Spadavecchia, J., Presicce, D.S., Taurino, A.M. (2003). Solid state gas sensors: state of the art and future activities. Journal of Optoelectronics and Advanced Materials, vol. 5, no. 5, p. 1335-1348.

[3] Das, T.K., and Prusty, S. (2012). Review on conducting polymers and their applications. Polymer-Plastics Technology and Engineering, vol. 51, no. 14, p. 1487-1500.

[4] Lei, W., Si, W., Xu, Y., Gu, Z., Hao, Q. (2014). Conducting polymer composites with graphene for use in chemical sensors and biosensors. Microchimica Acta. vol. 181, no. 7-8, p. 707-722.

[5] Liu, J., Lin, Y., Liang, L., Voigt, J.A., Huber, D.L., Tian, Z.R., Coker, E., Mckenzie, B., and Mcdermott, M.J. (2003). Templateless assembly of molecularly aligned conductive polymer nanowires: a new approach for oriented nanostructures. Chemistry-A European Journal, vol. 9, no. 3, p. 604-611.

[6] Matsubara, C., Kawamoto, N., and Takamura, K. (1992). Oxo [5, 10, 15, 20-tetra (4pyridyl) porphyrinato] titanium (IV): an ultra-high sensitivity spectrophotometric reagent for hydrogen peroxide. Analyst, vol. 117, no. 11, p. 1781-1784. 
[7] Nakashima, K., Maki, K., Kawaguchi, S., Akiyama, S., Tsukamoto, Y., and Imai, K. (1991). Peroxyoxalate Chemiluminescence Assay of Hydrogen Peroxide and Glucose Using 2, 4, 6, 8-Tetrathiomorpholinopyrimido (5, 4-d) pyrimidine as a Fluorescent Component. Analytical sciences, vol. 7, no. 5, p. 709-713.

[8] Song, Y., Wang, L., Ren, C., Zhu, G., and Li, Z. (2006). A novel hydrogen peroxide sensor based on horseradish peroxidase immobilized in DNA films on a gold electrode. Sensors and Actuators B: Chemical, vol. 114, no. 2, p. 1001-1006.

[9] Siao, H-W., Chen, S-M., and Lin, K-C. (2011). Electrochemical study of PEDOTPSS-MDB-modified electrode and its electrocatalytic sensing of hydrogen peroxide. Journal of Solid State Electrochemistry, vol. 15, no. 6, p. 1121-1128.

[10] Choi, J., Lee, J., Choi, J., Jung, D., Shim, S.E. (2010). Electrospun PEDOT: PSS/PVP nanofibers as the chemiresistor in chemical vapour sensing. Synthetic Metals, vol. 160, no. 13, p. 1415-1421.

[11] Yin, K. and Zhu, Z. (2010). "One-pot" synthesis, characterization, and $\mathrm{NH}_{3}$ sensing of Pd/PEDOT: PSS nanocomposite. Synthetic Metals, vol. 160, no. 9, p. 1115-1118.

[12] Pantalei, S., Zampetti, E., Bearzotti, A., Cesare, F.D., Macagnano, A. (2013). Improving sensing features of a nanocomposite PEDOT: PSS sensor for NO breath monitoring. Sensors and Actuators B: Chemical, vol. 179, p. 87-94.

[13] Li, Y., Hu, Y., Zhao, Y., Shi, G., Deng, L., Hou, Y., and Qu, L. (2011). An Electrochemical Avenue to Green-Luminescent Graphene Quantum Dots as Potential Electron-Acceptors for Photovoltaics. Advanced Materials, vol. 23, no. 6, p. 776-780.

[14] Kim, J.K., Park, M.J., Kim, S.J., Wang, D.H., Cho, S.P., Bae, S., Park, J.H., and Hong, B.H. (2013). Balancing light absorptivity and carrier conductivity of graphene quantum dots for high-efficiency bulk heterojunction solar cells. ACS nano, vol. 7, no. 8, p. 7207-7212.

[15] Gupta, V., Chaudhary, N., Srivastava, R., Sharma, G.D., Bhardwaj, R., and Chand, S. (2011). Luminscent graphene quantum dots for organic photovoltaic devices. Journal of the American Chemical Society, vol. 133, no. 26, p. 9960-9963.

[16] Kim, J.K., Kim, S.J., Park, M.J., Bae, S., Cho, S-P., Du, Q.G., Wang, D.H., Park, J.H., and Hong, B.H. (2015). Surface-Engineered Graphene Quantum Dots Incorporated into Polymer Layers for High Performance Organic Photovoltaics. Scientific reports, vol. 5.

[17] Van Tam, T., Trung, N.B., Kim, H.R., Chung, J.S., Choi, W.M. (2014). One-pot synthesis of $\mathrm{N}$-doped graphene quantum dots as a fluorescent sensing platform for $\mathrm{Fe}^{3+}$ ions detection. Sensors and Actuators B: Chemical, vol. 202: p. 568-573. 
[18] Gavgani, J.N., Dehsari, H.S., Hasani, A., Mahyari, M., Shalamzari, E.K., Salehi, A., and Taromi, F.A. (2015). A room temperature volatile organic compound sensor with enhanced performance, fast response and recovery based on $\mathrm{N}$-doped graphene quantum dots and poly (3, 4-ethylenedioxythiophene)-poly (styrenesulfonate) nanocomposite. $R S C$ Advances, vol. 5, no. 71, p. 57559-57567.

[19] Seekaew, Y., Lokavee, S., Phokharatkul, D., Wisitsoraat, A., Kerdcharoen, T., Wongchoosuk, C. (2014). Low-cost and flexible printed graphene-PEDOT: PSS gas sensor for ammonia detection. Organic Electronics, vol. 15, no. 11, p. 2971-2981.

[20] Taccola, S., Greco, F., Zucca, A., Innocenti, C., Fernández, Cd.J., Campo, G., Sangregorio, C., Mazzolai, B., and Mattoli, V. (2013). Characterization of free-standing PEDOT: PSS/iron oxide nanoparticle composite thin films and application as conformable humidity sensors. ACS applied materials \& interfaces, vol. 5, no. 13, p. 6324-6332.

[21] Mabrook, M.F., Pearson, C., and Petty, M.C. (2005). An inkjet-printed chemical fuse. Applied Physics Letters, vol. 86, no. 1, p. 013507.

[22] Mabrook, M.F., Pearson, C., and Petty, M.C. (2006). Inkjet-printed polymer films for the detection of organic vapors. Sensors Journal, IEEE, vol. 6, no. 6, p. 1435-1444.

[23] Zheng, Y., Lee, D., Koo, H.Y., and Maeng, S. (2015). Chemically modified graphene/PEDOT: PSS nanocomposite films for hydrogen gas sensing. Carbon, vol. 81: p. 54-62.

[24] Al-Mashat, L., Shin, K., Kalantar-zadeh, K., Plessis, J.D., Han, S.H., Kojima, R.W., Kaner, R.B., Li, D., Gou, X., Ippolito, S.J., and Wlodarski, W. (2010). Graphene/polyaniline nanocomposite for hydrogen sensing. The Journal of Physical Chemistry C, vol. 114, no. 39, p. $16168-16173$.

[25] Liu, J., Agarwal, M., and Varahramyan, K. (2008). Glucose sensor based on organic thin film transistor using glucose oxidase and conducting polymer. Sensors and Actuators B: Chemical, vol. 135, no. 1, p. 195-199.

[26] Wu, Z., Chen, X., Zhu, S., Zhou, Z., Yao, Y., Quan, W., and Liu, B. (2013). Room temperature methane sensor based on graphene nanosheets/polyaniline nanocomposite thin film. Sensors Journal, IEEE, vol. 13, no. 2, p. 777-782.

[27] Wu, Z., Chen, X., Zhu, S., Zhou, Z., Yao, Y., Quan, W., Liu, B. (2013). Enhanced sensitivity of ammonia sensor using graphene/polyaniline nanocomposite. Sensors and Actuators B: Chemical, vol. 178: p. 485-493. 
Bulletin de la Société Royale des Sciences de Liège, Vol. 85, 2016, p. 261 - 268

[28] Shim, Y.B., Stilwell, D.E., and Park, S.M. (1991). Electrochemistry of conductive polymers X: Polyaniline-based potentiometric sensor for dissolved oxygen. Electroanalysis, vol. 3, no. 1, p. 31-36. 